\title{
A Pilot Trial of Molecularly Tailored Therapy for Patients with Metastatic Pancreatic Ductal Adenocarcinoma
}

Anteneh A. Tesfaye, ${ }^{1}$ Hongkun Wang, ${ }^{2}$ Marion L. Hartley, ${ }^{2}$ Aiwu Ruth $\mathrm{He}_{1}{ }^{2}$ Louis Weiner, ${ }^{2}$ Nina Gabelia, ${ }^{2}$ Lana Kapanadze, ${ }^{2}$ Muhammad Shezad, ${ }^{2}$ Jonathan R. Brody, ${ }^{3}$ John L. Marshall, ${ }^{2}$ and Michael J. Pishvaian,**

\begin{abstract}
Purpose: Despite the wide adoption of tumor molecular profiling, there is a dearth of evidence linking molecular biomarkers for treatment selection to prediction of treatment outcomes in patients with metastatic pancreatic cancer. We initiated a pilot study to test the feasibility of designing a larger phase II trial of molecularly tailored treatment for metastatic pancreatic cancer.

Methods: Our study aimed to assess the feasibility of following a treatment algorithm based on the expression of three published predictive markers of response to chemotherapy: ribonucleotide reductase catalytic subunit M1 (for gemcitabine); excision repair cross-complementation group 1 (for platinum agents); and thymidylate synthase (for 5-fluorouracil) in patients with untreated, metastatic pancreatic cancer. Results of the tumor biopsy analysis were used to assign patients to one of seven doublet regimens. Key secondary objectives included response rate (RR), disease control rate (DCR), progression-free survival (PFS), and overall survival (OS).

Results: Between December 2012 and March 2015, 30 patients were enrolled into the study. Ten patients failed screening primarily due to inadequate tumor tissue availability. Of the remaining 20 patients, 19 were assigned into 6 different chemotherapy doublets, and achieved an RR of $28 \%$, with a DCR rate of $78 \%$. The median PFS and OS were 5.78 and 8.21 months, respectively.

Conclusions: The incorporation of biomarkers into a treatment algorithm is feasible and resulted in a PFS and OS similar to other doublet therapies for patients with metastatic pancreatic cancer. Based on the results from this pilot study, a larger phase II randomized trial of molecularly targeted therapy versus physicians' choice of standard of care has been initiated in the second-line setting (NCT02967770).
\end{abstract}

Keywords: chemotherapy; metastatic pancreatic cancer; molecular testing

\section{Introduction}

Pancreatic cancer is the third leading cause of cancerrelated mortality, and it is estimated that 53,670 people were diagnosed with, and 43,090 people died from this disease in 2018. Surgical resection is currently the only potentially curative treatment option, but unfortunately, only $9 \%$ and $29 \%$ of patients have operable or localized, nonmetastatic disease, respectively. The vast majority of patients are diagnosed with metastatic dis- ease on initial presentation, and these patients have a reported median survival of only $8-11$ months. ${ }^{1-5}$

Chemotherapy continues to be the cornerstone of treatment for pancreatic cancer since the approval of gemcitabine in the frontline setting. ${ }^{2}$ Modern day chemotherapy combinations have since improved outcomes over single agent gemcitabine, thereby becoming the new standards of care. In 2011, the combination of 5-fluorouracil (5FU), oxaliplatin, and irinotecan (FOLFIRINOX) was shown

\footnotetext{
${ }^{1}$ Karmanos Cancer Institute, Wayne State University, Detroit, Michigan.

${ }^{2}$ Lombardi Comprehensive Cancer Center, Georgetown University, Washington, District of Columbia.

${ }^{3}$ Department of Surgery, Sidney Kimmel Cancer Center, Thomas Jefferson University, Philadelphia, Pennsylvania.

*Address correspondence to: Michael J. Pishvaian, MD, PhD, Lombardi Comprehensive Cancer Center, Georgetown University, Podium B, 3800 Reservoir Road NW, Washington, DC 20007, E-mail: pishvaim@georgetown.edu
}

(C) Anteneh A. Tesfaye et al. 2019; Published by Mary Ann Liebert, Inc. This Open Access article is distributed under the terms of the Creative Commons License (http://creativecommons.org/licenses/by/4.0), which permits unrestricted use, distribution, and reproduction in any medium, provided the original work is properly cited. 
to improve median overall survival (OS) to 11.1 months compared with gemcitabine, which demonstrated a median OS of just 6.8 months in patients with a good performance status (ECOG 0-1). ${ }^{3}$ In 2013, the combination of gemcitabine and nab-paclitaxel was also shown to be better than single agent gemcitabine. The median OS of gemcitabine and nab-paclitaxel was 8.5 months, which was statistically superior to the 6.7-month survival seen with gemcitabine. ${ }^{6}$ These chemotherapy regimens in current clinical practice were evaluated empirically in nonbiomarker-enriched patient populations, and there are no accompanying predictive tools to guide their use in patients. Given the short-lived benefit from chemotherapy, it would be a worthwhile endeavor to select patients who are most likely to benefit from a given treatment while sparing treatment-related side effects for those who are less likely to benefit. Although the predictive strength of any single biomarker is debatable, ${ }^{7}$ the utility of a composite of multiple biomarkers in patient selection to match them with best available treatment option(s) has not been widely explored.

Despite the availability of molecular profiling, there is scarcity of high-quality prospective data evaluating the efficacy of linking molecular profiles to specific treatment choices in patients with pancreatic adenocarcinoma. Studies have explored the predictive role of some biomarkers to specific chemotherapeutic agents. One such biomarker is ribonucleotide reductase catalytic subunit M1 (RRM1) expression, which is a potential marker for gemcitabine resistance. ${ }^{8}$ In addition, the expression of excision repair cross-complementation group 1 (ERCC1) and thymidylate synthase (TS) may be markers for platinum resistance ${ }^{9,10}$ and 5FU resistance, ${ }^{7,11,12}$ respectively. We have recently performed a review of the value of these predictive biomarkers across disease types, and our findings suggest that there is value at a minimum to exploring the utilization of these biomarkers in clinical trials for patients with pancreatic and other cancer types. ${ }^{13}$ Examples of the incidence of high or low expression of RRM1, ERCC1, and TS have been previously published. For example, for RRM1, Valsecchi et al. detailed that, of 93 patients assessed, RRM1 expression by immunohistochemistry (IHC) was low in $61(65 \%)$ and high in 32 patients $(35 \%) .{ }^{14}$ Other smaller data sets reveal high expression of RRM1 to be observed in $34-50 \%$ of patient samples. ${ }^{15-17}$ For ERCC1, Valsecchi et al. detailed that, of 94 patients assessed, ERCC1 expression by IHC was low in $41(44 \%)$ and high in 53 patients $(56 \%) .{ }^{14}$ Fareed et al. and Hwang et al. detailed that high levels of
ERCC1 expression were observed in $\sim 50 \%$ of patient samples. ${ }^{18,19}$ Finally, for TS, Hu et al. evaluated pancreatic tumor tissue from 132 resected patients and determined that TS expression was high in 83 of $132(63 \%)$ and low in 49 of 132 patients (37\%). ${ }^{20}$ Formentini et al. revealed that TS expression in 130 pancreatic cancer patients was low in $56 \%$ and high in $43 \%$ of patients. ${ }^{21}$

We designed a pilot study to explore the feasibility of incorporating the use of predictive biomarkers RRM1, ERCC1, and TS to select therapeutic "doublet" chemotherapy for patients with metastatic pancreatic cancer. As per protocol, the stated primary objective was "to determine the estimates of outcomes necessary to plan and conduct subsequent studies with molecularly tailored therapy, for patients with metastatic pancreatic cancer." More practically stated, the primary objective was essentially to assess the feasibility of testing these markers in newly diagnosed patients, and incorporating the test results into the treatment-decision process. Our key secondary end-points, including disease control rate (DCR), time to progression, and OS, were preliminary assessed to determine the feasibility and efficacy of our "molecularly tailored therapy" selections.

\section{Patients and Methods}

Patient eligibility

Eligible patients were $\geq 18$ years of age, with cytologically/histologically confirmed metastatic pancreatic adenocarcinoma that was amenable to biopsy to obtain sufficient tissue for molecular profiling. Patients must have not received prior systemic therapy for metastatic disease, have measurable disease by RECIST version 1.1, an Eastern Cooperative Oncology Group (ECOG) performance status of $0-2$, and acceptable liver, renal, and hematologic laboratory values. The study protocol (NCT01888978) was approved by the local institutional review board, and all patients provided written informed consent.

\section{Study design and procedures}

This was an open-label pilot study designed to assess the feasibility of following a simple algorithm to treat patients with metastatic pancreatic cancer with a chemotherapy regimen based on three published predictive markers of response/resistance to chemotherapy. As per protocol, the primary objective was "to determine the estimates of outcomes necessary to plan and conduct subsequent studies with molecularly tailored therapy, for patients with metastatic pancreatic cancer." Biopsies of accessible metastatic lesions were performed 
by an interventional radiologist under standard biopsy procedures. The specimen was sent to Caris Life Sciences (Caris) for molecular analysis to be checked for expression of, at minimum, RRM1, ERCC1, and TS, although a broader panel was typically assessed. IHC was used to measure the expression of the markers, as per standard operating procedures internal to Caris. For each IHC, the staining intensity and the percent of positive cells was pro- vided by Caris, and the cutoff of "high" versus "low" was determined by Caris, using their internal database. These cutoff values did not change significantly in the course of this study. Patients with inadequate biopsy specimens who were unwilling to undergo a second biopsy were considered screen failures. Patients were assigned a treatment doublet based on expression of RRM1, ERCC1, and TS, using the algorithm depicted below (Fig. 1).
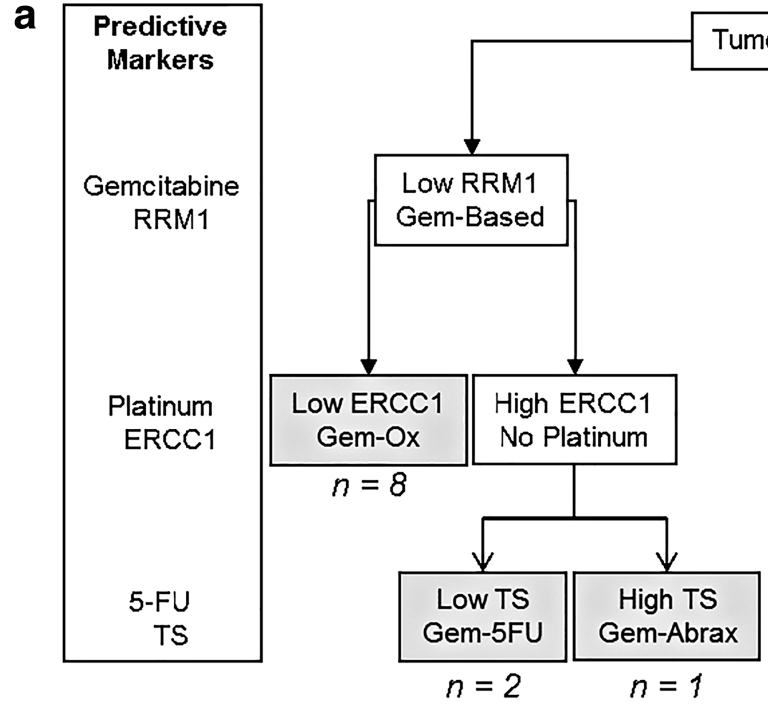

Tumor Biopsy

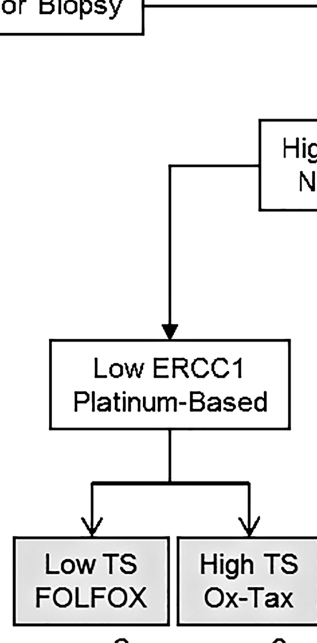

$n=3 \quad n=0$ 


\section{Treatment details}

Patients were treated with one of seven possible chemotherapy doublets, based on their tumor molecular profile (Table 1). Gem-Ox consisted of the combination of $1000 \mathrm{mg} / \mathrm{m}^{2}$ gemcitabine on day 1 and $100 \mathrm{mg} / \mathrm{m}^{2}$ oxaliplatin on day 2 , both given every 14 days, ${ }^{4,22-24}$ and was selected for patients with low RRM1 and ERCC1 expression. Gem-5FU consisted of the combination of $1000 \mathrm{mg} / \mathrm{m}^{2}$ gemcitabine and $2000 \mathrm{mg} / \mathrm{m}^{2}$ $5 \mathrm{FU}$, given as a $24-\mathrm{h}$ slow infusion, both administered on days 1,8 , and 15 of a 28 -day cycle, ${ }^{1,25-27}$ and was selected for patients with low RRM1, high ERCC1, and low TS expression. Gem-Abrax consisted of the combination of $1000 \mathrm{mg} / \mathrm{m}^{2}$ gemcitabine and $125 \mathrm{mg} / \mathrm{m}^{2}$ nabpaclitaxel, both given on days 1,8 , and 15 of a 28 -day cycle, ${ }^{5}$ and was selected for patients with low RRM1,

Table 1. Selection of Treatment Regimens Based on Tumor Molecular Profile

\begin{tabular}{|c|c|c|c|}
\hline Regimens & $\begin{array}{l}\text { Molecular } \\
\text { profile }\end{array}$ & $\begin{array}{c}\text { No. of } \\
\text { patients }\end{array}$ & Refs. \\
\hline $\begin{array}{l}\text { Gem-Ox } \\
\text { Gemcitabine } 1000 \mathrm{mg} / \mathrm{m}^{2} \text { day } 1 \\
\text { Oxaliplatin } 100 \mathrm{mg} / \mathrm{m}^{2} \text { day } 2 \\
\text { Every } 2 \text { weeks }\end{array}$ & $\begin{array}{l}\text { Low RRM1 } \\
\text { Low ERCC1 }\end{array}$ & 8 & $5,22-24$ \\
\hline $\begin{array}{l}\text { Gem-5FU } \\
\text { Gemcitabine } 1000 \mathrm{mg} / \mathrm{m}^{2} \\
5 \text {-FU } 2000 \mathrm{mg} / \mathrm{m}^{2} 24-\mathrm{h} \text { slow infusion } \\
\text { Both on days } 1,8 \text {, and } 15 \\
\text { of a } 28 \text {-day cycle }\end{array}$ & $\begin{array}{l}\text { Low RRM1 } \\
\text { High ERCC1 } \\
\text { Low TS }\end{array}$ & 2 & $2,25-27$ \\
\hline $\begin{array}{l}\text { Gem-Abrax } \\
\text { Gemcitabine } 1000 \mathrm{mg} / \mathrm{m}^{2} \\
\text { Nab-paclitaxel } 125 \mathrm{mg} / \mathrm{m}^{2} \\
\text { Both on days } 1,8, \text { and } 15 \\
\text { of a 28-day cycle }\end{array}$ & $\begin{array}{l}\text { Low RRM1 } \\
\text { High ERCC1 } \\
\text { High TS }\end{array}$ & 1 & 28 \\
\hline $\begin{array}{l}\text { FOLFOX } \\
\text { Oxaliplatin } 85 \mathrm{mg} / \mathrm{m}^{2} \text { day } 1 \\
\text { 5-Fluorouracil } 400 \mathrm{mg} / \mathrm{m}^{2} \text { day } 1 \\
\text { Leucovorin } 400 \mathrm{mg} / \mathrm{m}^{2} \text { day } 1 \\
\text { 5-Fluorouracil } 2400 \mathrm{mg} / \mathrm{m}^{2} \text { over } 46 \mathrm{~h} \\
\text { Every } 2 \text { weeks }\end{array}$ & $\begin{array}{l}\text { High RRM1 } \\
\text { Low ERCC1 } \\
\text { Low TS }\end{array}$ & 2 & $29-31$ \\
\hline $\begin{array}{l}\text { Ox-Tax } \\
\text { Oxaliplatin } 100 \mathrm{mg} / \mathrm{m}^{2} \text { day } 1 \\
\text { Docetaxel } 65 \mathrm{mg} / \mathrm{m}^{2} \text { day } 1 \\
\text { Mandatory growth factor support } \\
\text { Every } 3 \text { weeks }\end{array}$ & $\begin{array}{l}\text { High RRM1 } \\
\text { Low ERCC1 } \\
\text { High TS }\end{array}$ & 0 & 32 \\
\hline $\begin{array}{l}\text { FOLFIRI } \\
\text { Irinotecan } 180 \mathrm{mg} / \mathrm{m}^{2} \text { day } 1 \\
\text { Leucovorin } 400 \mathrm{mg} / \mathrm{m}^{2} \text { day } 1 \\
5 \text {-FU } 400 \mathrm{mg} / \mathrm{m}^{2} \text { day } 1 \\
5 \text {-FU } 2400 \mathrm{mg} / \mathrm{m}^{2} \text { over } 46 \mathrm{~h} \\
\text { Every } 2 \text { weeks }\end{array}$ & $\begin{array}{l}\text { High RRM1 } 1 \\
\text { High ERCC1 } \\
\text { Low TS }\end{array}$ & 4 & $33-36$ \\
\hline $\begin{array}{l}\text { Tax-Iri } \\
\text { Docetaxel } 35 \mathrm{mg} / \mathrm{m}^{2} \\
\text { Irinotecan } 50 \mathrm{mg} / \mathrm{m}^{2} \\
\text { Weekly for } 4 \text { of } 6 \text { weeks }\end{array}$ & $\begin{array}{l}\text { High RRM1 } \\
\text { High ERCC1 } \\
\text { High TS }\end{array}$ & 1 & 37,38 \\
\hline
\end{tabular}

5FU, 5-fluorouracil; ERCC1, excision repair cross-complementation group 1; RRM1, ribonucleotide reductase catalytic subunit M1; TS, thymidylate synthase. high ERCC1, and high TS expression. FOLFOX consisted of the combination of $85 \mathrm{mg} / \mathrm{m}^{2}$ oxaliplatin on day $1,400 \mathrm{mg} / \mathrm{m}^{2} 5 \mathrm{FU}$ on day $1,400 \mathrm{mg} / \mathrm{m}^{2}$ leucovorin on day 1 , and $2400 \mathrm{mg} / \mathrm{m}^{2} 5 \mathrm{FU}$ over $46 \mathrm{~h}$, all administered every 14 days, ${ }^{28-30}$ and was selected for patients with high RRM1, low ERCC1, and low TS expression. Ox-Tax consisted of the combination of $100 \mathrm{mg} / \mathrm{m}^{2}$ oxaliplatin on day 1 and $65 \mathrm{mg} / \mathrm{m}^{2}$ docetaxel on day 1 , both given every 3 weeks with growth factor support, ${ }^{31}$ and was selected for patients with high RRM1, low ERCC1, and high TS expression. 5FU/leucovorin and irinotecan (FOLFIRI) consisted of the combination of $180 \mathrm{mg} / \mathrm{m}^{2}$ irinotecan on day $1,400 \mathrm{mg} / \mathrm{m}^{2} 5 \mathrm{FU}$ on day 1 , leucovorin $400 \mathrm{mg} / \mathrm{m}^{2}$ on day 1 , and $5 \mathrm{FU} 2400 \mathrm{mg} / \mathrm{m}^{2}$ over $46 \mathrm{~h}$, all administered every 14 days, ${ }^{32-35}$ and was selected for patients with high RRM1, high ERCC1, and low TS expression. Finally, Tax-Iri consisted of the combination of $35 \mathrm{mg} / \mathrm{m}^{2}$ docetaxel and $50 \mathrm{mg} / \mathrm{m}^{2}$ irinotecan, both given weekly for 4 of 6 weeks, ${ }^{36,37}$ and was selected for patients with high RRM1, high ERCC1, and high TS expression. Dose modifications for adverse events were detailed in the protocol for each regimen. Treatment in the assigned subgroup was continued until disease progression or patient intolerance occurred, and all patients were followed until death.

\section{Statistical analysis}

As a pilot study, no specific statistical hypotheses were tested or were utilized in determining the "estimates of outcomes necessary to plan and conduct subsequent studies." Rather, descriptive summary statistics were used for patient demographics as well as toxicities $(N$, median, range for continuous variables; and $N$, percent for categorical variables). Kaplan-Meier methodology was used to estimate the progression-free survival (PFS) and OS. Median PFS and OS were presented with their 95\% confidence intervals. SAS 9.3 (SAS, Inc., Cary, NC) was used for the analysis.

\section{Results}

Between December 2012 and March 2015, 30 patients with metastatic pancreatic adenocarcinoma were consented, and 19 of these received molecularly tailored therapy as per protocol (Fig. 1a, b). The median age of patients was 63 years (range: 42-76). The majority of the patients were male $(63 \%)$ and Caucasian (68\%). Most patients (63\%) had ECOG performance status of 1 . The baseline demographics and diseaserelated characteristics of the patients are summarized in Table 2. 
Table 2. Patient Demographics

\begin{tabular}{lc}
\hline Characteristics & Enrolled patients $(\boldsymbol{n}=19)$ \\
\hline Age (in years) & \\
$\quad$ Median (range) & $63(42-76)$ \\
Gender, $N(\%)$ & $7(36.8)$ \\
$\quad$ Female & $12(63.2)$ \\
$\quad$ Male & \\
Race, $N(\%)$ & $2(10.5)$ \\
$\quad$ Asian & $4(21.1)$ \\
Black & $13(68.4)$ \\
$\quad$ White & \\
Disease stage, $N(\%)$ & $19(100.0)$ \\
$\quad$ IV & $5(26.3)$ \\
ECOG performance status, $N(\%)$ & $12(63.2)$ \\
0 & $2(10.5)$ \\
1 & \\
2 &
\end{tabular}

ECOG, Eastern Cooperative Oncology Group.

Of the 30 patients consented, 20 (67\%) had enough tissue available for tumor molecular profiling and consequent treatment assignment. One patient was a screen failure after biopsy due to biliary obstruction. The remaining 19 patients were able to initiate molecularly tailored therapy. The median time from biopsy to treatment initiation was 32.5 days (range: 14-68). The most common tumor molecular profile was low RRM1 and ERCC1 (44.4\%), and these patients were treated with a doublet of gemcitabine and oxaliplatin. The second-most commonly seen profile was high
RRM1, high ERCC1, and low TS (22.2\%), and these patients were treated with a doublet of FOLFIRI.

Patients continued therapy with protocol-defined dose modifications for adverse events until disease progression or intolerance. At the time of submission of this article, all patients had completed follow-up for PFS and OS, with the exception of one patient who moved out of the country and was lost to follow-up after 3 months.

The CA 19-9 best response is shown in Figure 2 (for the 15 patients with baseline elevation in CA 19-9), and $55 \%$ of patients had $>50 \%$ reduction in their tumor marker. The 6-month DCR was $78 \%$, with partial response and stable disease seen in $28 \%$ and $50 \%$ of patients, respectively. The median PFS was 5.78 months (95\% CI 5.39-15.72), while the median OS was 8.21 months (95\% CI 7.16-15.72). Treatment durations are depicted in Figure 3. Survival and response details are listed in Table 3, and PFS and OS are depicted in Figure $4 \mathrm{a}$ and $\mathrm{b}$.

Grade 3 adverse events reported among the patients treated with molecularly tailored regimens included nausea/vomiting (10\%), anemia (10\%), thrombocytopenia (10\%), venous thromboembolism (5\%), peripheral neuropathy (5\%), and febrile neutropenia (5\%). There were no Grade 4 adverse events reported (Table 4 for complete list of adverse events).

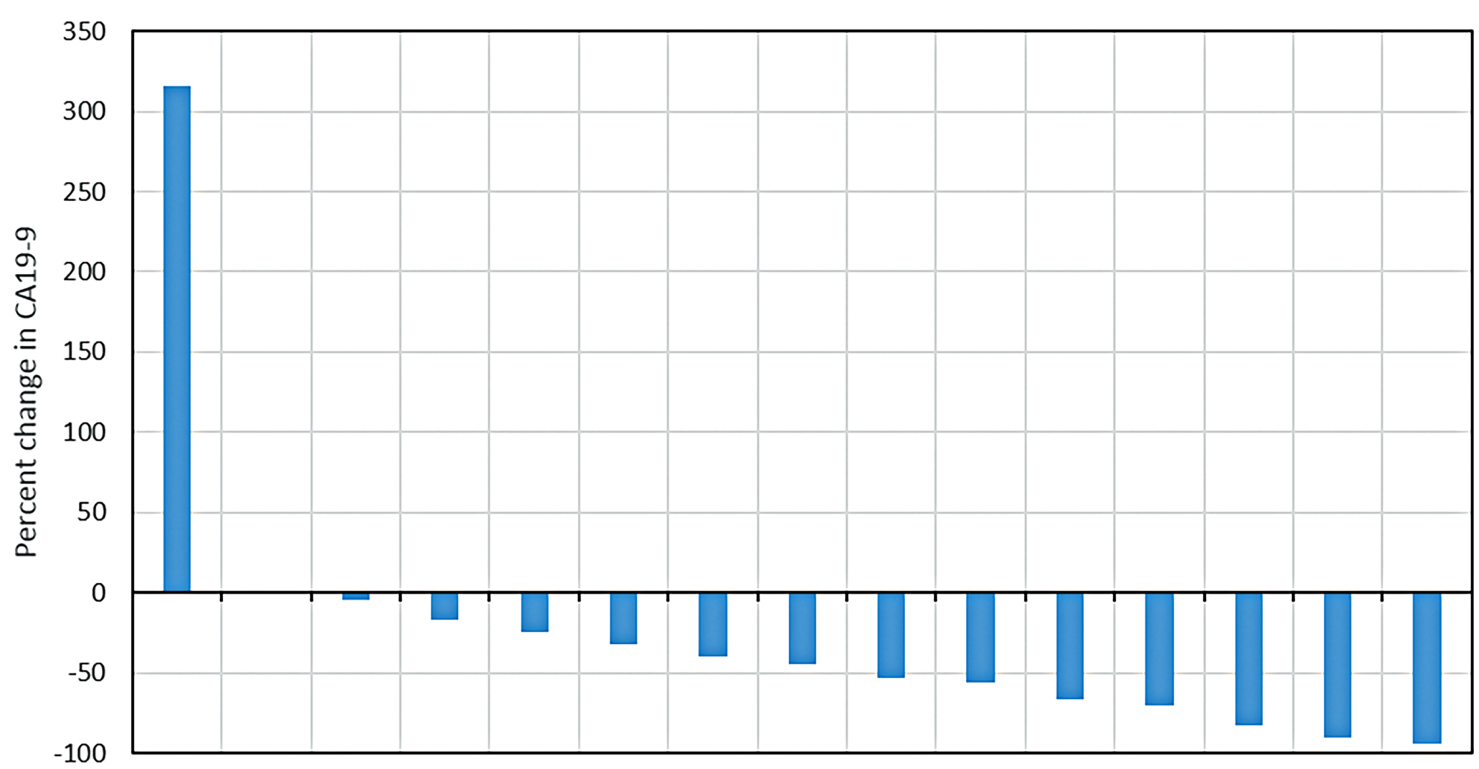

FIG. 2. Percent changes in CA19-9 from pretreatment level to the nadir reported on the study. 


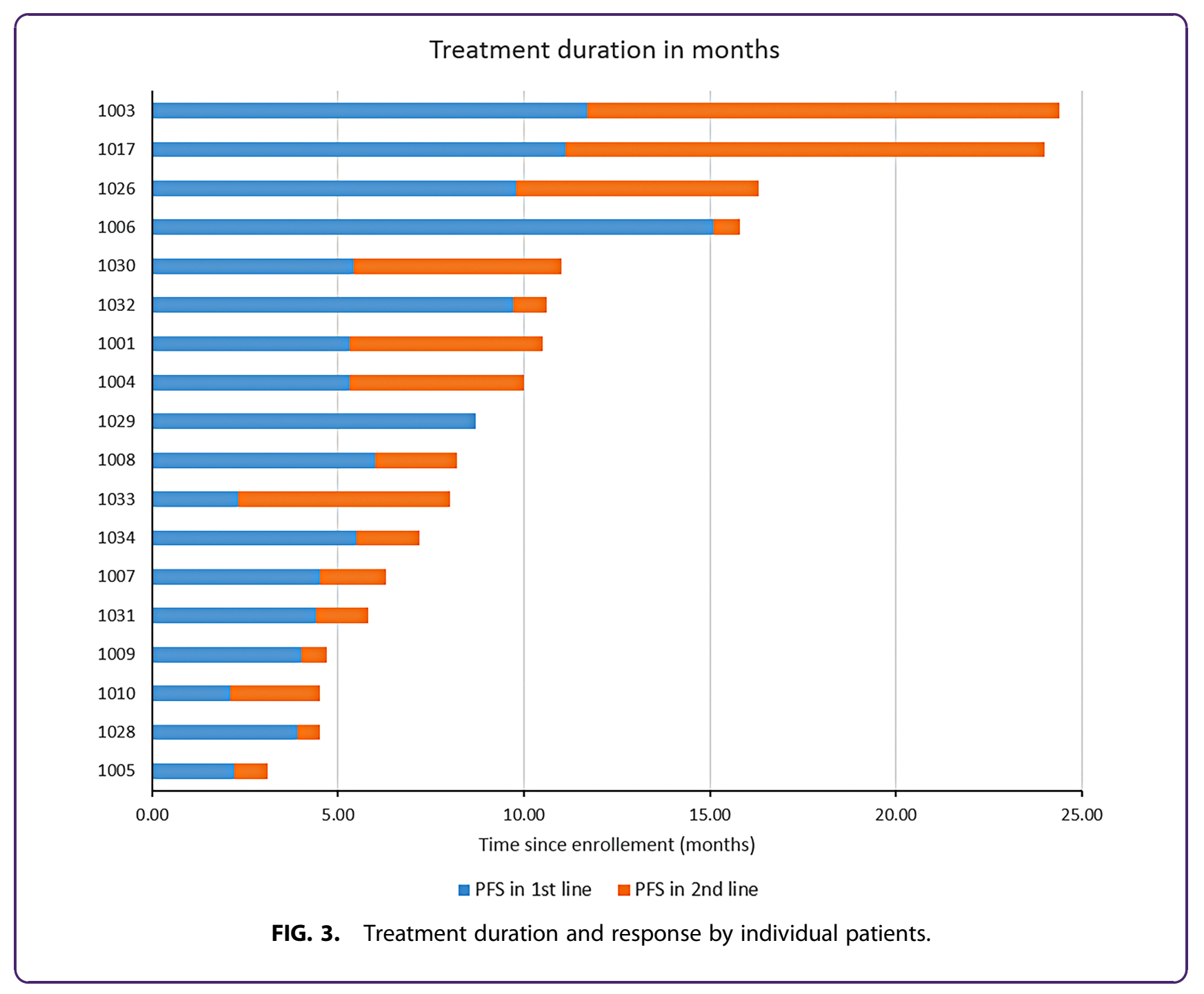

On disease progression, eight patients (40\%) did not receive any further treatment, of whom seven (88\%) died within 3 months of failure of the frontline therapy. Twelve patients (60\%) underwent secondline therapy at the discretion of the treating physician, of whom four patients (33\%) received gemcitabine and nab-paclitaxel, three patients (25\%) received FOLFOX/XELOX, two patients (16.7\%) received FOLFIRI, two patients $(16.7 \%)$ pursued further clinical trials (immunotherapy, capecitabine/ruxolitinib), and one patient (8\%) received single agent 5FU. Patients who had disease progression after frontline treatment but were able to receive second-line therapy had a median OS with further treatment of 5.4 (range: 0.7-13.4) months.

\section{Discussion}

Tailoring therapy to the molecular profiles of tumor Although we have greatly improved our understanding of the molecular etiology of pancreatic adenocarcinoma, this has not translated into clinically meaningful treatment options for patients. In fact, no personalized nor even targeted therapy has resulted in clinically meaningful improvements in patient outcomes. By contrast, novel cytotoxic chemotherapy regimens such as FOLFIRINOX and gemcitabine plus nab-paclitaxel have led to an increased OS, while maintaining a reasonable quality of life. ${ }^{3,5}$ These successes indicate that optimal utilization of cytotoxic agents, including gemcitabine, oxaliplatin, irinotecan, 5FU, and taxanes (nab-paclitaxel, paclitaxel, or docetaxel), can improve patient outcomes. 
Table 3. Maximum Response: RECIST, CA19-9, and Survival

\begin{tabular}{lc}
\hline Response & $\boldsymbol{N}(\%)$ \\
\hline RECIST best tumor response & \\
Disease control rate & $14(78)$ \\
Complete response & $0(0)$ \\
Partial response & $5(28)$ \\
Stable disease & $9(50)$ \\
Progressive disease & $4(22)$ \\
CA 19-9 reduction (total $n=15), n(\%)$ & \\
$>20 \%$ & $15(83)$ \\
$>50 \%$ & $10(55)$ \\
$>90 \%$ & $4(22)$ \\
Survival (months) (95\% Cl) & \\
PFS, months & $5.78(5.39-15.72)$ \\
OS, months & $8.21(7.16-15.72)$ \\
Alive at 6 months, $n(\%)$ & $13(72)$ \\
\hline
\end{tabular}

OS, overall survival; PFS, progression-free survival.

Unfortunately, the success of the various cytotoxic chemotherapy regimens is often diluted when tested in large, unselected patient populations. ${ }^{1-4,6,38,39}$ The inability to translate the significant benefits seen in some patients to the pancreatic cancer population at large is because, in common practice, promising chemotherapy

Table 4. All Cause Adverse Events

\begin{tabular}{lcl}
\hline Adverse events & All grades $(\boldsymbol{N})$ & Grade $\mathbf{3 , N}(\%)$ \\
\hline Nonhematologic AE & & \\
Fatigue & 13 & \\
Nausea/vomiting & 8 & $2(10)$ \\
Anorexia & 4 & \\
Hypoalbuminemia & 2 & \\
Weight loss & 2 & \\
Constipation & 2 & \\
Abdominal distention & 1 & \\
Edema & 2 & \\
Gastroesophageal reflux & 2 & \\
Paresthesia & 7 & $1(5)$ \\
Fever & 3 & \\
Alopecia & 2 & \\
Dehydration & 3 & \\
Hypokalemia & 2 & \\
Peripheral sensory neuropathy & 2 & \\
Renal calculi/renal colic & 1 & \\
Hematuria & 1 & \\
Hemorrhage & 1 & \\
Cough & 1 & \\
Hypoglycemia & 1 & \\
Hypotension & 1 & \\
Hematologic & & \\
Anemia & 4 & $2(10)$ \\
Platelet count decreased & 3 & $2(10)$ \\
Thromboembolic event & 2 & $1(5)$ \\
Febrile neutropenia & 1 & $1(5)$ \\
Wound infection & 1 & $1(5)$ \\
Catheter-related infection & 1 & \\
$\uparrow$ Alanine aminotransferase & 3 & $1(5)$ \\
$\uparrow$ Alkaline phosphatase & 3 & $1(5)$ \\
$\uparrow$ Aspartate aminotransferase & 1 & $1(5)$ \\
\hline
\end{tabular}

There were no Grade 4 adverse events reported. $\mathrm{AE}$, adverse events. combinations are never administered to select patients who are most likely to respond based on their tumor's molecular profile. However, more data have emerged recently ${ }^{40}$ defining molecular subgroups of patients with pancreatic cancer, who, if appropriately selected, could benefit from currently available therapies. This understanding makes a persuasive argument to enhance patient outcomes by adapting predictive tumor biomarkers that will provide a realistic platform for physicians to select treatment with better precision from an array of available agents and regimens for their patients. We initiated a pilot trial to assess the feasibility of tailoring established and FDA-approved cytotoxic chemotherapies for pancreatic cancer patients through molecular analyses of their tumors.

\section{Molecular targets and predictors}

Recognizing the low probability of a single-tumor biomarker to be predictive of chemotherapy benefit in general, we utilized an algorithm of several published markers with the intent of improving clinical outcomes compared with contemporary, nonbiomarker-enriched treatment strategies.

In this study, patients underwent fresh tumor biopsies and the tumors were assessed for three specific published predictive markers of response to chemotherapy. The first such marker is RRM1, which can predict resistance to gemcitabine. ${ }^{8}$ The overexpression of ERCC1 can lead to resistance to platinum drugs.,10 The expression of TS can predict resistance to $5 \mathrm{FU},{ }^{7,11,12}$ Based on the tumor biomarker profile, the algorithm outlined in Figure 1 was used to assign patients to one of seven possible "doublet" chemotherapy combinations. There are no high-quality prospective data that show a clinically meaningful benefit as a result of using these biomarkers to guide treatment selection. This pilot trial was a feasibility study to plan a larger phase II study of the use of such biomarkers to guide treatment selection for patients with advanced pancreatic cancers.

\section{Outcomes using molecular predictors}

to select therapy

Treatment offered to patients based on their molecular profiles was very well tolerated, although it is difficult to compare any specific adverse event to larger studies due to the small number of patients. This trial demonstrated the feasibility of obtaining and analyzing fresh tumor biopsies to guide treatment selection. However, there was a $40 \%$ screen failure rate, indicating the constraints of making treatment decisions based on the 

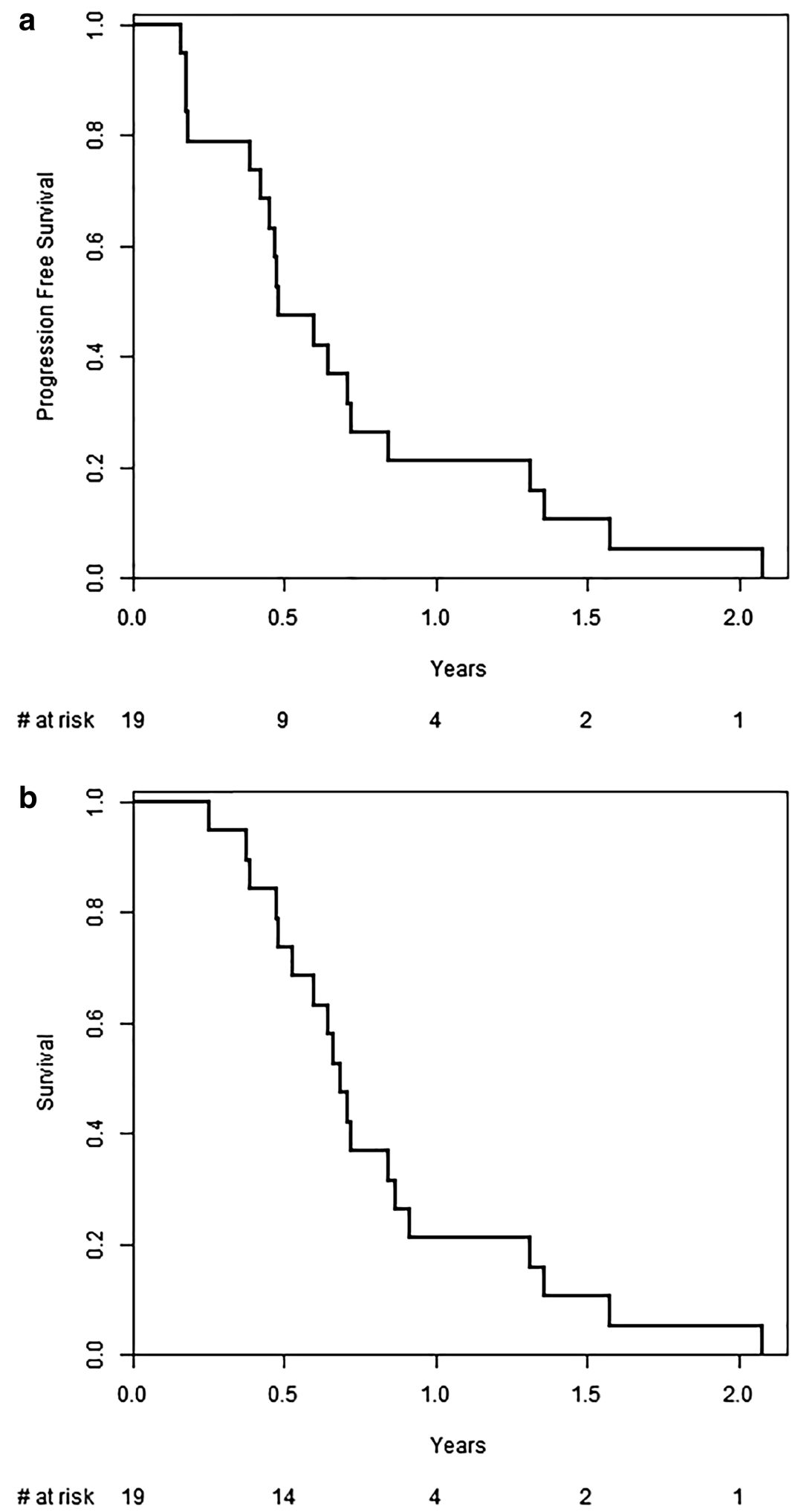

FIG. 4. Median PFS and OS. (a) Graph showing Kaplan-Meier of PFS. The median PFS ( $95 \% \mathrm{Cl}$ ) was 5.78 months (5.39-15.72). (b) Graph showing Kaplan-Meier of OS. The median OS (95\% Cl) was 8.21 months (7.16-15.72). Cl, confidence interval; OS, overall survival; PFS, progression-free survival. 
molecular profile of tumors in previously untreated patients who are likely to have significant disease-related symptoms and may not be able to afford waiting for tumor profiling results before a treatment decision can be made and therapy is initiated.

Moreover, our data suggest a potential benefit to molecularly tailored therapy, with the majority of patients achieving a 6-month DCR of 78\%, with partial response and stable disease seen in $28 \%$ and $50 \%$ of patients, respectively. The majority (55\%) of patients had $>50 \%$ reduction in their tumor marker (CA 19-9). The median PFS of 5.78 months (95\% CI 5.39-15.72) and the median OS of 8.21 months (95\% CI 7.16-15.72) were similar to other published doublet therapy results. ${ }^{1,2,438}$

\section{Second-line treatment outcomes}

A significant portion (40\%) of the study population was not able to pursue further treatment for their disease. This underscores the need for effective frontline therapy to potentially benefit these patients, a significant proportion of whom will never be able to pursue treatment in the second-line setting once the frontline regimen fails. These patients may potentially benefit from molecularly tailored treatment strategies, which may help identify regimens that are likely/unlikely to be effective. Contrary to the treatment outcomes in the frontline setting, the effectiveness of chemotherapy beyond the frontline setting sharply declines in patients with metastatic pancreatic carcinoma. Second-line trials historically have shown very low response rates (less than $30 \%$ ) and OS of 4-6 months. ${ }^{41-48}$ Although the sample was small, our study's findings are in line with those previously described. ${ }^{41-48}$

\section{Conclusions}

The incorporation of biomarkers to guide the selection of chemotherapy is feasible and resulted in a similar PFS and OS compared with other standard therapies for patients with metastatic pancreatic cancer. To investigate the benefit of using this approach, a randomized trial versus standard of care has been developed in the second-line setting, taking into consideration the high rate of screen failures due to inadequate tissue sampling (NCT02967770).

\section{Acknowledgments}

M.J.P. and J.R.B. are supported by a 2015 Pancreatic Cancer Action Network American Association for Cancer Research Acceleration Network Grant (15-9025-BROD). The trial was funded by The Ruesch Center for the Cure of Gastrointestinal Cancers at Lombardi Comprehensive Cancer Center.

\section{Author Disclosure Statement}

No competing financial interests exist.

\section{References}

1. Berlin JD, Catalano P, Thomas JP, et al. Phase III study of gemcitabine in combination with fluorouracil versus gemcitabine alone in patients with advanced pancreatic carcinoma: Eastern Cooperative Oncology Group Trial E2297. J Clin Oncol. 2002;20:3270-3275.

2. Burris HA, III, Moore MJ, Andersen J, et al. Improvements in survival and clinical benefit with gemcitabine as first-line therapy for patients with advanced pancreas cancer: a randomized trial. J Clin Oncol. 1997;15: 2403-2413.

3. Conroy $T$, Desseigne F, Ychou M, et al. FOLFIRINOX versus gemcitabine for metastatic pancreatic cancer. N Engl J Med. 2011;364:1817-1825.

4. Poplin E, Feng Y, Berlin J, et al. Phase III, randomized study of gemcitabine and oxaliplatin versus gemcitabine (fixed-dose rate infusion) compared with gemcitabine (30-minute infusion) in patients with pancreatic carcinoma E6201: a trial of the Eastern Cooperative Oncology Group. J Clin Oncol. 2009;27:3778-3785.

5. Von Hoff DD, Ervin T, Arena FP, et al. Increased survival in pancreatic cancer with nab-paclitaxel plus gemcitabine. N Engl J Med. 2013;369: 1691-1703.

6. Von Hoff DD, Ramanathan RK, Borad MJ, et al. Gemcitabine plus nab-paclitaxel is an active regimen in patients with advanced pancreatic cancer: a phase I/II trial. J Clin Oncol. 2011;29:4548-4554.

7. Grimminger PP, Shi M, Barrett $C$, et al. TS and ERCC- 1 mRNA expressions and clinical outcome in patients with metastatic colon cancer in CONFIRM-1 and -2 clinical trials. Pharmacogenomics J. 2012;12:404-411.

8. Xie $\mathrm{H}$, Jiang $\mathrm{W}$, Jiang J, et al. Predictive and prognostic roles of ribonucleotide reductase $\mathrm{M} 1$ in resectable pancreatic adenocarcinoma. Cancer. 2013;119:173-181

9. Heinemann V, von Weikersthal LF, Decker T, et al. FOLFIRI plus cetuximab versus FOLFIRI plus bevacizumab as first-line treatment for patients with metastatic colorectal cancer (FIRE-3): a randomised, open-label, phase 3 trial. Lancet Oncol. 2014;15:1065-1075.

10. Li P, Fang YJ, Li F, et al. ERCC1, defective mismatch repair status as predictive biomarkers of survival for stage III colon cancer patients receiving oxaliplatin-based adjuvant chemotherapy. Br J Cancer. 2013;108: 1238-1244.

11. Larsson PA, Carlsson G, Gustavsson B, et al. Thymidylate synthase in advanced gastrointestinal and breast cancers. Acta Oncol. 1996;35:469-472.

12. Kumamoto K, Kuwabara K, Tajima Y, et al. Thymidylate synthase and thymidine phosphorylase mRNA expression in primary lesions using laser capture microdissection is useful for prediction of the efficacy of FOLFOX treatment in colorectal cancer patients with liver metastasis. Oncol Lett. 2012;3:983-989.

13. Rao S, Beckman RA, Riazi S, et al. Quantification and expert evaluation of evidence for chemopredictive biomarkers to personalize cancer treatment. Oncotarget. 2016;8:37923-37934.

14. Valsecchi $M E$, Holdbrook $T$, Leiby $B E$, et al. Is there a role for the quantification of RRM1 and ERCC1 expression in pancreatic ductal adenocarcinoma? BMC Cancer. 2012;12:104.

15. Ashida R, Nakata B, Shigekawa $M$, et al. Gemcitabine sensitivity-related mRNA expression in endoscopic ultrasound-guided fine-needle aspiration biopsy of unresectable pancreatic cancer. J Exp Clin Cancer Res. 2009;28:83.

16. Jordheim LP, Seve $P$, Tredan $O$, et al. The ribonucleotide reductase large subunit (RRM1) as a predictive factor in patients with cancer. Lancet Oncol. 2011;12:693-702.

17. Akita H, Zheng Z, Takeda Y, et al. Significance of RRM1 and ERCC1 expression in resectable pancreatic adenocarcinoma. Oncogene. 2009;28: 2903-2909.

18. Fareed KR, Al-Attar A, Soomro IN, et al. Tumour regression and ERCC1 nuclear protein expression predict clinical outcome in patients with gastro-oesophageal cancer treated with neoadjuvant chemotherapy. $\mathrm{Br} \mathrm{J}$ Cancer. 2010;102:1600-1607. 
19. Hwang IG, Jang JS, Do JH, et al. Different relation between ERCC1 overexpression and treatment outcomes of two platinum agents in advanced biliary tract adenocarcinoma patients. Cancer Chemother Pharmacol. 2011;68:935-944.

20. Hu YC, Komorowski RA, Graewin S, et al. Thymidylate synthase expression predicts the response to 5-fluorouracil-based adjuvant therapy in pancreatic cancer. Clin Cancer Res. 2003;9:4165-4171.

21. Formentini A, Sander S, Denzer S, et al. Thymidylate synthase expression in resectable and unresectable pancreatic cancer: role as predictive or prognostic marker? Int J Colorectal Dis. 2007;22:49-55.

22. Airoldi M, Cattel L, Passera R, et al. Gemcitabine and oxaliplatin in patients with pancreatic adenocarcinoma: clinical and pharmacokinetic data. Pancreas. 2006;32:44-50.

23. Lee $\mathrm{KH}$, Kim MK, Kim YH, et al. Gemcitabine and oxaliplatin combination as first-line treatment for advanced pancreatic cancer: a multicenter phase II study. Cancer Chemother Pharmacol. 2009;64:317-325.

24. Louvet C, Labianca R, Hammel P, et al. Gemcitabine in combination with oxaliplatin compared with gemcitabine alone in locally advanced or metastatic pancreatic cancer: results of a GERCOR and GISCAD phase III trial. J Clin Oncol. 2005;23:3509-3516.

25. Di Costanzo F, Carlini P, Doni L, et al. Gemcitabine with or without continuous infusion 5-FU in advanced pancreatic cancer: a randomised phase II trial of the Italian oncology group for clinical research (GOIRC). $\mathrm{Br}$ J Cancer. 2005;93:185-189.

26. Louvet C, Andre T, Hammel P, et al. Phase II trial of bimonthly leucovorin, 5-fluorouracil and gemcitabine for advanced pancreatic adenocarcinoma (FOLFUGEM). Ann Oncol. 2001;12:675-679.

27. Santasusana JM, Garcia Lopez JL, Garcia JJ, et al. A phase II trial of gemcitabine and weekly high-dose 5 -fluorouracil in a 48-hour continuousinfusion schedule in patients with advanced pancreatic carcinoma. A study of the Spanish Cooperative Group for Gastrointestinal Tumour Therapy (TTD). Clin Transl Oncol. 2005;7:493-498.

28. Boeck S, Hoehler T, Seipelt G, et al. Capecitabine plus oxaliplatin (CapOx) versus capecitabine plus gemcitabine (CapGem) versus gemcitabine plus oxaliplatin (mGemOx): final results of a multicenter randomized phase II trial in advanced pancreatic cancer. Ann Oncol. 2008;19:340-347.

29. Ghosn M, Farhat F, Kattan J, et al. FOLFOX-6 combination as the first-line treatment of locally advanced and/or metastatic pancreatic cancer. Am J Clin Oncol. 2007;30:15-20.

30. Novarino A, Satolli MA, Chiappino I, et al. Oxaliplatin, 5-fluorouracil, and leucovorin as second-line treatment for advanced pancreatic cancer. Am J Clin Oncol. 2009;32:44-48.

31. Kim JG, Sohn SK, Chae YS, et al. Multicenter phase II study of docetaxel plus oxaliplatin combination chemotherapy in patients with advanced gastric cancer: Daegu Gyeongbuk Oncology Group. Br J Cancer. 2008;98:542-546.

32. Gebbia V, Maiello E, Giuliani F, et al. Irinotecan plus bolus/infusional 5Fluorouracil and leucovorin in patients with pretreated advanced pancreatic carcinoma: a multicenter experience of the Gruppo Oncologico Italia Meridionale. Am J Clin Oncol. 2010;33:461-464.

33. Neuzillet $C$, Hentic $O$, Rousseau $B$, et al. FOLFIRI regimen in metastatic pancreatic adenocarcinoma resistant to gemcitabine and platinum-salts. World J Gastroenterol. 2012;18:4533-4541.

34. Taieb J, Lecomte T, Aparicio T, et al. FOLFIRI.3, a new regimen combining 5-fluorouracil, folinic acid and irinotecan, for advanced pancreatic cancer: results of an Association des Gastro-Enterologues Oncologues (Gastroenterologist Oncologist Association) multicenter phase II study. Ann Oncol. 2007;18:498-503.

35. Yoo C, Hwang JY, Kim JE, et al. A randomised phase II study of modified FOLFIRI.3 vs modified FOLFOX as second-line therapy in patients with gemcitabine-refractory advanced pancreatic cancer. Br J Cancer. 2009; 101:1658-1663.

36. Bleickardt E, Argiris A, Rich R, et al. Phase I dose escalation trial of weekly docetaxel plus irinotecan in patients with advanced cancer. Cancer Biol Ther. 2002;1:646-651.

37. Burtness B, Thomas $L$, Sipples R, et al. Phase II trial of weekly docetaxel/ irinotecan combination in advanced pancreatic cancer. Cancer J. 2007;13: 257-262.
38. Herrmann R, Bodoky G, Ruhstaller T, et al. Gemcitabine plus capecitabine compared with gemcitabine alone in advanced pancreatic cancer: a randomized, multicenter, phase III trial of the Swiss Group for Clinical Cancer Research and the Central European Cooperative Oncology Group. J Clin Oncol. 2007;25:2212-2217.

39. Paez D, Labonte MJ, Lenz HJ. Pancreatic cancer: medical management (novel chemotherapeutics). Gastroenterol Clin North Am. 2012;41:189209.

40. Sjoquist KM, Chin VT, Chantrill LA, et al. Personalising pancreas cancer treatment: when tissue is the issue. World J Gastroenterol. 2014;20:78497863.

41. Assaf $E$, Verlinde-Carvalho $M$, Delbaldo $C$, et al. 5-fluorouracil/leucovorin combined with irinotecan and oxaliplatin (FOLFIRINOX) as second-line chemotherapy in patients with metastatic pancreatic adenocarcinoma. Oncology. 2011;80:301-306.

42. Cantore M, Rabbi C, Fiorentini G, et al. Combined irinotecan and oxaliplatin in patients with advanced pre-treated pancreatic cancer. Oncology. 2004;67:93-97.

43. Cereda S, Reni M, Rognone A, et al. Salvage therapy with mitomycin and ifosfamide in patients with gemcitabine-resistant metastatic pancreatic cancer: a phase II trial. Chemotherapy. 2011;57:156-161.

44. Chen L-T, Von Hoff DD, Li C-P, et al. Expanded analyses of napoli-1: Phase 3 study of MM-398 (nal-IRI), with or without 5-fluorouracil and leucovorin versus 5-fluorouracil and leucovorin, in metastatic pancreatic cancer (mPAC) previously treated with gemcitabine-based therapy. ASCO Meeting Abstracts. 2015;33:234.

45. Demols A, Peeters M, Polus M, et al. Gemcitabine and oxaliplatin (GEMOX) in gemcitabine refractory advanced pancreatic adenocarcinoma: a phase II study. Br J Cancer. 2006;94:481-485.

46. Oettle H, Riess H, Stieler JM, et al. Second-line oxaliplatin, folinic acid, and fluorouracil versus folinic acid and fluorouracil alone for gemcitabinerefractory pancreatic cancer: outcomes from the CONKO-003 trial. J Clin Oncol. 2014;32:2423-2429.

47. Tsavaris N, Kosmas C, Skopelitis $\mathrm{H}$, et al. Second-line treatment with oxaliplatin, leucovorin and 5-fluorouracil in gemcitabine-pretreated advanced pancreatic cancer: a phase II study. Invest New Drugs. 2005;23: 369-375

48. Xiong HQ, Varadhachary GR, Blais JC, et al. Phase 2 trial of oxaliplatin plus capecitabine (XELOX) as second-line therapy for patients with advanced pancreatic cancer. Cancer. 2008;113:2046-2052.

Cite this article as: Tesfaye AA, Wang $H$, Hartley $M L$, He $A R$, Weiner $L$ Gabelia N, Kapanadze L, Shezad M, Brody JR, Marshall JL, Pishvaian M (2019) A pilot trial of molecularly tailored therapy for patients with metastatic pancreatic ductal adenocarcinoma, Journal of Pancreatic Cancer 5:1, 12-21, DOI: 10.1089/pancan.2019.0003.

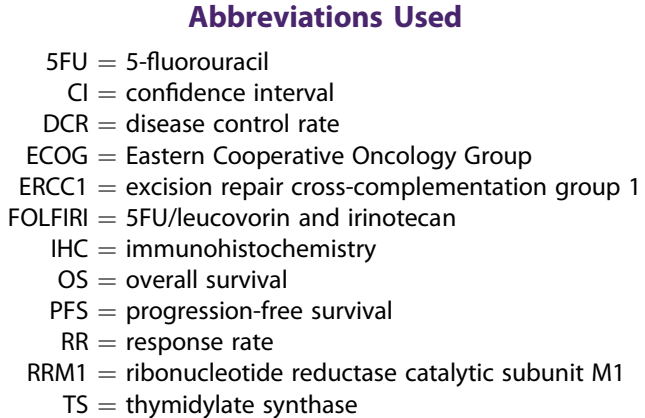

Abbreviations Used

$5 \mathrm{FU}=5$-fluorouraci

$\mathrm{Cl}=$ confidence interval

$\mathrm{DCR}=$ disease control rate

ECOG $=$ Eastern Cooperative Oncology Group

ERCC 1 = excision repair cross-complementation group 1

FOLFIRI $=5 \mathrm{FU} /$ leucovorin and irinotecan

$\mathrm{IHC}=$ immunohistochemistry

OS = overall survival

$\mathrm{PFS}=$ progression-free survival

$\mathrm{RR}=$ response rate

RRM1 = ribonucleotide reductase catalytic subunit M1

TS $=$ thymidylate synthase 